\title{
Boire en eaux troubles
}

La construction de la défiance et des risques de boire de l'eau

Drinking troubled waters. The setting up of distrust and risk about drinking water

\section{Céline Hervé-Bazin}

\section{OpenEdition \\ Journals}

Édition électronique

URL : http://journals.openedition.org/communicationorganisation/4534

DOI : 10.4000/communicationorganisation.4534

ISSN : $1775-3546$

\section{Éditeur}

Presses universitaires de Bordeaux

\section{Édition imprimée}

Date de publication : 1 juin 2014

Pagination : 127-138

ISBN : 978-2-86781-904-9

ISSN : $1168-5549$

\section{Référence électronique}

Céline Hervé-Bazin, «Boire en eaux troubles », Communication et organisation [En ligne], 45 | 2014, mis en ligne le 01 juin 2017, consulté le 30 avril 2019. URL : http://journals.openedition.org/ communicationorganisation/4534; DOI : 10.4000/communicationorganisation.4534 


\title{
Boire en eaux troubles \\ La construction de la défiance et des risques de boire de l'eau
}

\author{
Céline Hervé-Bazin'
}

«Qualité de l'eau, à qui faire confiance ? ». En mars 2013, l'association 60 millions de consommateurs révèle l'existence de traces de pesticides et de médicaments dans les eaux minérales. Scandale sanitaire récurrent, les controverses sur la qualité de l'eau de boisson sont inscrites dans le paysage médiatique français. Des nitrates en Bretagne aux pesticides, de l'aluminium aux médicaments, les scandales sur l'eau à boire se répètent mêlant des enjeux de santé publique, d'expertise ou encore, de confiance. Tous ont en commun de bâtir des discours sur la notion de risque au quotidien, de participer à la manufacture des risques (Beck, 2001) en insistant sur le double caractère dangereux et bienfaisant de l'eau pour la santé.

La confiance et défiance des Français au sujet de l'eau à boire font l'objet de baromètres annuels et d'actualités médiatiques répétées. Les traces de pollution dans l'eau du robinet et les bouteilles d'eau minérale participent d'un débat médiatique sur les ressources en eau. En parallèle, le Centre d'information sur l'eau (CIEAU) publie un baromètre annuel avec TNS Sofres sur les perceptions et la consommation de l'eau des Français depuis 17 ans. Selon le dernier rapport produit en 2013, les Français se déclarent satisfaits de la qualité de l'eau de leur domicile. Cet indicateur a progressé depuis 2007, soit $5 \%$ sur 5 ans. Ils sont $78 \%$ à renouveler leur confiance dans l'eau du robinet ou $83 \%$ à adopter une consommation responsable. Ils sont enfin, inquiets et sceptiques face à l'avenir des ressources en eau : $90 \%$ estiment qu'elle est limitée et rare. Cette confiance annoncée contraste avec les controverses médiatiques et querelles scientifiques, avec la consommation de

1 Céline Hervé-Bazin est Docteure en Sciences de l'Information et de la Communication à l'Université Paris Sorbonne / CELSA. Elle est chercheure au GRIPIC, EA 1498. Elle travaille notamment sur les thématiques environnementales et en particulier, sur l'eau et le changement climatique ainsi que sur les enjeux liés au genre ; celinehervebazin@gmail.com

Céline Hervé-Bazin holds a Ph D in Information and Communication Sciences from Sorbonne University and the CELSA, a high-level communication school. She is researcher at the GRIPIC, EA 1498. She works on environmental issues in particular, on water resources and climate change. She is also specialized on gender issues; celinehervebazin@gmail.com 
l'eau minérale qui reste forte et interroge avec les pratiques de consommation de boissons sucrées au détriment de l'eau.

Les débats, expertises et contre-expertises, jouent sur les paradoxes et la complexité du cycle de production de l'eau du robinet et de l'eau minérale où le consommateur aurait besoin d'être rassuré sur la protection et les dangers éventuels pour sa santé. Ces attaques sur l'eau à boire révèlent d'un processus de construction des discours rebondissant sur fonds de «scandales » sur lesquels les entreprises vont établir des stratégies de marque créant une complexité volontaire pour bâtir une avec leurs cibles.

Les discours de l'eau à boire ont alors pour but de conforter des consommateurs qui «boivent en eaux troubles ». Les stratégies établies reflètent la permanence des discours des organisations de l'eau face à des habitudes de consommation et des perceptions des Français qui évolueraient. Au fil de ces discours, l'eau à boire établit des argumentaires proches de convictions politiques voire idéologiques érigeant une opposition entre des "partisans ", d'un côté, les consommateurs de l'eau du robinet et de l'autre, ceux de l'eau minérale. Ces conceptions qui se confrontent, nourrissent le discours construit d'une guerre de l'eau (N'Guyen, 2004). À l'heure où les ressources en douce sont fragilisées et mobilisées, nous nous intéressons sur les impacts de ces discours sur la consommation d'eau du robinet et de l'eau minérale. Dans quelle mesure « la guerre de l'eau " publique et médiatique a réduit l'acte de boire a un acte de consommation banal qui permet d'appartenir à un groupe, de s'identifier et de retrouver des valeurs comme pour n'importe quel produit marketing ? En quoi les controverses qui font « boire en eaux troubles " ne sont-elles pas une menace directe sur les ressources en eau et le bien être des consommateurs? En érigeant l'acte de boire comme un acte dangereux, deux phénomènes sont nés : la surconsommation des boissons sucrées à la place de l'eau et boire revient à adhérer à un groupe de valeurs divisant les citoyens là où l'eau est la ressource universelle liant les individus entre eux.

Nous avons étudié cinq grands scandales de l'eau de boisson en France à partir d'une analyse de discours de 40 articles de presse publiés entre 1998 et 2013. Nous avons également constitué un corpus de 20 campagnes de communication des entreprises d'eau minérale (Evian, Contrex, Cristaline et le syndicat des eaux minérales) et des entreprises en charge de la production et distribution d'eau du robinet (Syndicat des eaux d'Ile de France - SEDIF, les villes de Besançon et Mulhouse, Eau de Paris, la Fédération des professionnels de l'eau - Fp2e).

Pour illustrer notre propos, nous aborderons tout d'abord, comment le discours sur l'eau à boire est construit autour du danger sanitaire intégré à la fabrique du risque (Beck, 2001). Nous montrerons ensuite souligner la répétition des registres facilitant la mise en scène d'une querelle d'expertise ont institué un rituel de prises de parole sur l'espace public et médiatique. Ce rituel répète des discours et des valeurs qui s'intègrent à l'imaginaire collectif 
et au contrat social. En dernière partie, nous montrerons en quoi ces débats continus opposent des conceptions de vie à travers la manière de boire de l'eau. Ces conceptions participent à une identité collective et individuelle qui mettent en danger l'acte de boire de l'eau et les liens sociaux entre les individus. Ces discours participent d'un éco-blanchiment, un «blue washing » qui met en scène des rites de communication, des marques et des discours qui contredisent l'idéal universel de la protection des ressources en eau et du slogan « l'eau source de vie ».

\section{L'eau à boire, construction des risques et des oppositions}

$\mathrm{Au}$ cours des quinze dernières années, plusieurs scandales médiatiques ont éclaté en France mettant sur le devant de la scène, les enjeux liés à la qualité de l'eau du robinet. Le premier concerne la présence d'aluminium dans l'eau du robinet dans un dossier publié en 1998 par France Soir. En 2001, les cas de légionellose bouleversent le paysage médiatique. Entre 2004 et 2005, la querelle oppose la ville de Paris et les services d'eau du robinet à la marque Cristaline. En 2008, c'est le scandale de l'eau et le cancer ; il sera associé à celui de la maladie d'Alzheimer qui suggère un lien entre boire de l'eau du robinet et cette maladie. En 2010, le documentaire Du poison dans l'eau diffusé sur France 3 ravive les controverses. Enfin, en 2013, une étude alerte sur les pesticides et médicaments présents dans les bouteilles d'eau minérale.

Pour chaque scandale, la révélation d'une nuisance est portée dans la presse par trois types de figures emblématiques : le journaliste $(1998,2010)$, l'association et l'expert (2001, 2008 et 2013), et enfin, l'entreprise (2004). La prise de parole est souvent simplifiée autour de déclarations alarmantes : "Aluminium attention danger " (1998) ; "Scandales des tuyaux rouillés " (2001) ; "Malades du cancer, ne buvez pas l'eau du robinet » ou encore " Maladie d'Alzheimer : l'eau du robinet en cause » (2008) ; "La France spécialiste des scandales sanitaires » ou « L'eau du robinet en cause » (2010); et enfin, "Qui croire ? Notre santé est-elle menacée par notre eau » (2013).

L'analyse des articles de presse révèle la récurrence lexicale du registre sanitaire (maladie, contamination, risque sanitaire, etc.) représentant 15,5\% du corpus analysé. Les enjeux de santé se déclinent autour de maladies médiatiques symboliques (légionnelle, cancer, Alzheimer) ou de pollutions, sujet tout aussi emblématique (nitrates, aluminium, amiante, médicaments, hormones). Le thème de la pollution compte pour $19,5 \%$ du corpus et désigne le plus souvent un risque pour la santé de l'individu. Peu porte sur la pollution des ressources à l'exception des zones de captage qui alimentent les usines de production d'eau potable. D'autres éléments portent sur le lexique de la recherche (étude, analyse, enquête...) ou un langage technique et médical précis décrivant les maladies. Autour, ce sont les acteurs impliqués qui jouent un rôle important dans l'information apportée.

La part représentée par les acteurs est révélatrice de la mise en scène des 
discours de chacun. Constituant $13 \%$ du corpus, nous avons comptabilisé 93 acteurs différents issus des ministères, les institutions publiques, des experts et des praticiens de la santé, des journalistes, la réglementation, les minéraliers et les distributeurs de service privé. Dans ce cortège de discours, le mot " risque " apparaît 68 fois (sur un total de 4189 mots analysés). Il est associé à un ensemble constitué de mots comme "controverse ", "sonnette d'alarme ", " nocif », «scandale ", " polémique " qui nourrissent " la fabrique de risques" (U. Beck).

Dans le corpus étudié, certains publics cibles sont privilégiés pour alerter et nourrir la controverse médiatique comme les populations vulnérables :

«Les femmes enceintes et les nourrissons doivent s'abstenir d'en consommer. » (L'Expansion, $1^{\text {er }}$ janvier 2008)

«On fait attention à l'eau que l'on donne aux bébés parce qu'on sait qu'ils ont une santé fragile. Eh bien, on doit tenir le même raisonnement pour les personnes malades ou âgées. » (Le Parisien, 26 janvier 2009)

Les enjeux sont également alarmistes, centrés sur des propos inquiétants :

"Si vous êtes atteint d'un cancer, méfiez-vous de l'eau du robinet ", c'est ce qu'explique en substance le rapport rédigé sous la direction du WWF et de David ServanSchreiber (médecin psychiatre), responsable du site Guérir.fr, qui réunit une communauté de personnes victimes de cette maladie. L'équipe signant le texte comprend essentiellement des médecins, dont Luc Montagnier, prix Nobel 2008. » (Futura Sciences, 2009)

«Il prétend, résultats à l'appui, qu'il a l'arme chimique contre cette bactérie tueuse qui prolifère dans les réseaux de canalisation d'eau chaude, dans ceux des climatisations ou encore dans les tours aéro-réfrigérantes. » (Tarn \& Garonne, 2001)

Le ton et le langage alternent des verbes affirmatifs, induisant des ordres (devoir, interdiction) avec du conditionnel accompagné de mots induisant le doute, l'inquiétude ou la menace (sonnette d'alarme, s'inquiéter, prévenir, précaution). Dans ce dispositif lexical, les paroles laissent place à des querelles de position où certains affirment catégoriquement, d'autres induisent des doutes. Le risque est construit à partir de plusieurs discours, acteurs et contextes, ce qui accélère et accentue son potentiel de nuisance pour l'individu.

Selon C. Kermisch (2012, conclusion, paragraphe 53) la définition et la compréhension du risque doit être "une définition du risque ouverte et contextuelle, capable de rendre compte de la complexité et de la spécificité de chaque type de risque dans le contexte où il se développe (...), l'intérêt d'une telle définition réside dans la réhabilitation des valeurs et des critères qualitatifs dans un cadre d'analyse plus global. » C. Kermisch montre que cette double dimension participe d'une notion de risque fondamentalement construite et dépendant de représentations quantitatives et qualitatives. Cette perception du risque qui dépend d'un contexte où la complexité s'introduit, est favorisée 
par un éclatement des prises de parole qui alimentent des oppositions entre différentes formes de figures occupant l'espace public et médiatique.

\section{L'eau à boire, la bataille des prises de parole}

Les discours sur l'eau de boisson sont complexes, diffusés autour d'une lexicographie confuse qui les rendent difficiles à comprendre en particulier sur les aspects techniques (Macpherson, Slovic : 2010 ; Simpson, Stratton : 2011) et jouant avec les perceptions du public (Gleick, 2011). Ces discours se sont généralisés et résumés pour être diffusés de l'échelle globale à locale, sur l'espace médiatique auprès de tous les publics et de toutes les organisations afin d'obtenir des consensus et de rassembler autour de lieux communs et d'idéaux partagés (D'Almeida, 2007). Le but ultime est de rassurer le consommateur sur l'eau qu'il boit à travers la promesse d'une eau saine et sans danger pour la santé. L'eau à boire doit être l'eau parfaite, pure et transparente. L'enjeu discursif est de vouloir réduire, synthétiser et réunir autour de représentations sociales partagées. Il s'agit d'alimenter la construction de stéréotypes du discours sur l'eau à boire pour le rattacher à un mythe fondamental, un inconscient de l'eau de boisson (Bachelard, 1942).

Ces principales représentations rejoignent différents imaginaires communs que partage notre société comme celui de la transparence, de la menace sanitaire grave (contamination de l'eau qui traduit une nouvelle forme de guerre), de l'invisibilité des menaces environnementales (identifiée par D. Bourg sur les discours environnementaux) ou encore, de la confiance. En particulier pour l'eau de boisson, c'est sa pureté qui est au cœur des axes de communication des différentes campagnes de communication et des scandales alimentaires. Après le scandale de la viande de cheval, les révélations de 60 millions de consommateurs insistent sur la soi-disant " pureté » des eaux en bouteille.

"Ces eaux, en effet, ne sont pas jugées pour leur seule qualité sanitaire, qui doit être une évidence, mais aussi pour leur "pureté originelle». » (Le Figaro, 25 mars 2013)

«La quantité de produits détectée est suffisante pour qu'on s'interroge sur la pureté originelle imposée par la règlementation des eaux minérales. » (Elle, décembre 2013)

"Mais ces traces montrent que certaines eaux en bouteille ne sont pas si pures que cela!» (RTL, 25 mars 2013)

Le trouble jeté dans les esprits favorise l'exploitation de ces mythes collectifs pour livrer la guerre des mots et des prises de position. Chacun affirme son rôle et s'insurge contre son opposant.

"Au nom de la liberté d'expression, et du droit des consommateurs à se faire eux-mêmes leur propre opinion, on peut penser que la récente condamnation de la société Cristaline, est tout à fait partiale. Dans le sens où les mêmes qui ont porté plainte contre cette société dépassent chaque jour largement la mesure sur leurs sites Internet, beaux ouvrages de propagande, d'appel au boycott de l'eau en bouteille, de 
dénigrement, et de désinformation, s'agissant de notre secteur (...) On se croirait revenu au temps de l'inquisition. " (Syndicat des eaux de sources, 2005 suite au scandale Cristaline)

«L'enquête «ne met absolument pas en cause l'honnêteté des embouteilleurs», mais interroge la contamination de l'environnement par les pratiques humaines, ajoute-t-il. «Il y a inquiétude sur la qualité de la ressource globale», résume Thomas Laurenceau » (WWF, 2013 suite au scandale des médicaments dans l'eau)

Dans notre corpus, les discours opposent les experts entre eux sur le sujet des scandales sanitaires; les entreprises sur l'eau en bouteille et robinet se divisent sur la qualité, la proximité et le marketing ; ou encore, les associations s'affrontent l'État sur les normes et réglementations sur la qualité de l'eau. Les débats portent sur le bien fondé des affirmations d'experts accélérant l'exploitation de ces « peurs irrationnelles » selon Michel Desmars et Clotilde Carron de la Fédération Nationale des Collectivités Concédantes et Régies (FNCCR) cités par Franck Gintrand (Crises Corporate, 2010).

Ces controverses s'appuient sur un processus de diffusion des scandales sur l'eau reprenant des éléments redondants : l'événement déclencheur, le lexique d'opposition, les figures prenant parole (expert, association, journaliste), la vulgarisation des propos et la publicisation des débats, et enfin, l'établissement de perceptions. Dans cette relation science-médias-public, la confrontation devient discursive, nourrie d'incertitudes qui sont divulguées, répétées et s'instituent comme des rituels codés semblant conforter les attitudes des consommateurs à travers des représentations sociales basées sur les notions de risque, en particulier la santé (qualité de l'eau à boire) et l'environnement (qualité des eaux de sources et zones de captage).

Par exemple, en 2008, le médecin David Servan-Schreiber publie avec le Fond Mondial pour la protection de la Nature (WWF), un rapport établissant les liens entre les personnes souffrant de cancer, personnes âgées ou personnes fragiles physiquement et les dangers que l'eau du robinet peut représenter pour leur santé dans le cadre de leurs maladies. En moins de quelques jours, l'information est transformée et diffusée largement dans les médias : l'eau du robinet peut causer le cancer. La diffusion de l'annonce a été reprise, raccourcie et amplifiée dans un phénomène qui rend public une vérité scientifique fausse. En conséquence, un vif débat est lancé et le docteur Servan-Schreiber est obligé de spécifier ses propos. Ce dernier précise et invite à la modération; il s'agit de comprendre que les personnes vulnérables ne devraient pas boire d'eau du robinet qui présenterait davantage de risques que l'eau en bouteille.

Une telle construction du processus de diffusion est similaire dans les autres scandales étudiés où les processus des prises de parole se répètent. L'accélération de l'information brute qui alerte devient rapidement un nouveau «scandale ", une polémique qui inquiète et laisse les consommateurs perplexes. La construction de ces prises de parole qui s'affrontent permet 
l'exploitation d'un registre de valeur destiné à rassurer. Les acteurs présents sur la sphère publique construisent leurs campagnes de communication sur ces querelles médiatiques afin de promouvoir des valeurs individuelles et de société dans le simple fait de choisir sa manière de boire.

\section{L'eau à boire, valeurs et manière d'être}

Les minéralités sont connues pour utiliser les meilleurs mécanismes de communication pour vendre leurs produits aux consommateurs. Nombre de recherches ont déjà analysé les différents éléments bâtissant leur campagne, le plus souvent étant empruntés à l'imaginaire commun et jouant sur les peurs des consommateurs vis-à-vis de l'eau du robinet. Evian est connu pour avoir exploité la valeur de la jeunesse rappelant le mythe de jouvence mais investissant en même temps celle de l'enfant et de l'exigence de la qualité de l'eau donnée aux bébés. Remises en question pour la véracité des vertus qu'elles promettent, elles ont dû revoir leur communication pour entrer en conformité avec les principes d'une communication responsable qui informe sans mentir sur les qualités réelles des produits vendus. Un des exemples les plus récents de ce changement discursif est celui opéré par la marque Contrex.

La campagne de publicité de la marque Contrexintitulée «Contrexpérience», lancée en 2011, a permis de redessiner les contours de la marque glissant des valeurs de " minceur » à celle du sport et de l'alimentation équilibrée. Le slogan de la marque change, du célèbre « contrat minceur » à " partenaire minceur ». Dans cette logique discursive, Contrex bâtie sa nouvelle campagne sur $\mathrm{Ma}$ Contrexpérience dont le choix du slogan est révélateur, la « contre - expérience ». Cette campagne de communication permet un changement discursif tout en préservant l'identité de la marque. Dans les années 90, le message s'est basé sur l'eau de "Contrex fait à mincir » aux années 2000 à «Contrex vous accompagne dans votre régime alimentaire ». Ces glissements soulignent la capacité à manier les discours pour incarner des valeurs. Le bébé d'Evian présent dès les premières campagnes au début du $\mathrm{XX}^{\mathrm{e}}$ siècle est un symbole particulièrement parlant. La dernière publicité Evian "Baby and me " rappelle la promesse de la marque qui joue sur le mythe de l'eau de jouvence. La marque investit alors la valeur de la jeunesse en alliant bébés et personnes âgées ou encore, le slogan : « Evian, source de jeunesse ».

Ces campagnes contribuent à nourrir des imaginaires profonds et inconscients de l'eau, source de vie, de plaisir, et de bonne santé. Les eaux minérales garantissent une eau de qualité et bâtissent leur exploitation commerciale sur une notion d'accès à une certaine idée de l'eau, un imaginaire de l'eau qui s'écarte du lexique du danger et des peurs collectives. L'eau des minéraliers devient l'eau qui permet d'accéder à des valeurs supérieures (jeunesse, beauté, terroir, etc.). Leur stratégie évite les scandales alimentaires en s'adressant directement aux valeurs de l'individu. Dans cette guerre des discours sur l'eau, l'eau du robinet a peu à peu investi le terrain du marketing 
grand public pour promouvoir des nouvelles formes de boire l'eau notamment autour des notions de citoyenneté, proximité, accessibilité et individualité responsable et engagée.

Au cours des 10 dernières années, l'eau du robinet est devenue un lieu de bataille marketing. En France, de nombreuses villes se sont engagées pour promouvoir l'eau du robinet en particulier, à partir de 2004. Le Syndicat des Eaux d'Ile de France a lancé une campagne de publicité pour promouvoir l'eau du robinet en utilisant des étiquettes reproduisant le format des eaux en bouteille. Les quatre slogans proposent des arguments connus de registres de la communication environnementale. En 2005, la ville de Paris est la première à distribuer gratuitement, au cours d'une large communication événementielle, des carafes en France. Lévénement est organisé sur le parvis de la Mairie de Paris et largement repris par les médias. Simultanément, la Fp2e lance une grande campagne de sensibilisation sur l'eau du robinet intitulée « Merci l'eau du robinet ». Cet effet nouveauté reprend des initiatives déjà lancées notamment aux Pays-Bas en 2003. L'agence de communication Vandejong, basée à Amsterdam, lance une bouteille d'eau du robinet appelée « Neau ". P. Gleick (2010) considère l'expérience néerlandaise comme la première du genre ouvrant la guerre au marketing contre l'eau en bouteilles.

Cette brèche marketing s'accélère. À Besançon, la ville décide de produire sa propre eau du robinet, la Bisontine, qu'elle vend dans les supermarchés locaux. À Mulhouse, la ville lance une bouteille créée par un artiste pour promouvoir la ville pendant le prestigieux festival d'Art Basel pour attirer les touristes. Aujourd'hui, la plupart des grandes villes ont leurs carafes comme Dijon, Lille, Marseille... Outil marketing qui diffuse l'image de la ville à travers un objet du quotidien qui s'introduit dans les maisons, les cafés, les restaurants.

En parallèle de ce mouvement, plusieurs entrepreneurs décident de commercialiser des bouteilles d'eau du robinet remettant la gourde au goût du jour. En France, c'est Gobi. Première éco-bouteille qui s'adresse au public et aux entreprises ou encore, en Angleterre, Edwin Broni-Mensah, doctorant, lance " Give me tap » ( Apporte-moi un robinet»), bouteille destinée aux étudiants qui investit rapidement la capitale anglaise. Toutes ces initiatives s'accompagnent d'applications Internet et proposent une carte des fontaines à eau dans la ville. Cette communication ancrée dans le territoire, réintègre les pratiques de consommation d'eau autour de la fontaine, point de rencontres et de socialisation (Caulier, 1990). L'eau du robinet distribuée en public initie une nouvelle forme de vie collective qui réinvestit celle de la fontaine à travers des opérations de marketing territorial comme Eau de Paris en 2012. En partenariat avec Instagram, Eau de Paris déploie une série de visuels simples et efficaces pour créer la connivence avec leur public comme "Après le selfie, le self'eau !». Il s'agit de prendre sa photo devant une fontaine de Paris. L'individu, citoyen habitant la ville de Paris, est interpellé et expose sa 
personnalité et ses convictions au nom de l'eau de sa ville.

Ces campagnes instrumentalisent une guerre de l'eau autour de valeurs de l'individu. Interrogé dans une ère de l'incertitude, de scandales alimentaires, les discours mettent en scène des controverses pour faire réagir sur les convictions personnelles. Ces confusions ramènent à des choix fondamentalement personnels qui se font au détriment de l'intérêt collectif malgré les efforts de rationalisation des discours notamment portés par les experts, eux-mêmes, divisés, doutant ou affirmant mais n'offrant plus de réponses tangibles si ce n'est celle du jugement personnel. Alors que slogan consensuel « l'eau, c'est la vie » donne l'impression qu'il y a partage, l'eau interpelle autour d'une multiplicité de croyances, d'expertises, et du lien fondamental que chaque individu entretient avec les ressources en eau.

\section{Conclusion}

La circulation des discours participe à des disputes, des expertises qui se confrontent et la construction de perceptions où le risque au quotidien alimente des représentations et les comportements des individus vis-à-vis de l'eau qu'ils boivent. Le débat public et la participation des individus sont intégrés à la co-construction d'un espace public où sont interrogées les valeurs du lien social incarnées par l'eau, ressource universelle et vitale.

Cette instrumentalisation des risques, des scandales et des expertises qui se contredisent, bâtissent la manufacture des risques et celles de valeurs personnelles confortant un style de vie, un système de croyances et une manière de percevoir la société. Ces controverses jettent le trouble dans la gestion des ressources en eau pourtant capitale et défavorisent la consommation de l'eau qu'elle soit du robinet ou minérale favorisant la consommation des boissons sucrées néfastes pour la santé.

Autre limite, ces discours établissent des modalités de comprendre, d'analyser et d'agir similaires qui classent, rassurent et divisent en groupes d'utilisateurs devenus des individus engagés sans pour autant rassembler. Cette manufacture des discours empêche l'acceptation des risques réels, la nécessité d'un changement de(s) comportement(s) au service du bien-être collectif, du contrat social et de la préservation de l'eau.

\section{BIBLIOGRAPHIE}

BACHELARD G., L'eau et les rêves, essai sur l'imagination de la matière, Librairie José Corti, Paris, France, 1942.

BECK U., La société du risque, Coll. Alto, éd. Aubier, Paris, France, 2001.

CANS R., La Bataille de l'eau, ré-édition 1997, Broché, Paris, France, 1994.

CALLON M. et LASCOUMES P., BARTHES Y., Agir dans un monde incertain, Paris, Le Seuil, 2001. 
CAULIER B., «L'eau et le sacré. Les cultes thérapeutiques autour des fontaines en France du Moyen-Âge à nos jours. » Presses de l'Université de Laval, Beauchesne, Canada, 1997.

CHARAUDEAU P., Les médias et l'information. L'impossible transparence du discours, De Broeck \& Larcier, Bruxelles, Belgique, 2005.

CHATEAURAYNAUD F., Argumenter dans un champ de forces. Essai de balistique sociologique, Éditions PETRA, Paris, France, 2011.

DEMOULIERE R. et BENSAID SCHEMBA J., BERGER J., AÏT KACI S., ROUGIE F., Les services d'eau et d'assainissement en France, Rapport BIPE / Fp2e, 5 édition, France, 2012.

GLEICK P., Bottled and Sold: The Story Behind Our Obsession with Bottled Water, Island Press, Washington D.C., USA, 2011.

HERVE-BAZIN C., Water Communication. Analysis of Strategies and Campaigns from the Water Sector, IWA Publishing, London, U.K., 2014.

IFOP, Les entreprises et l'eau : perceptions, comportements et gestion, IFOP pour Hydrelis, Paris, France, 2011.

MACPHERSON L., SLOVIC P., Talking about Water. Vocabulary and Images that Support Informed Decisions about Water Recycling and Desalination, WateReuse Research, Foundation Alexandria, USA, 2011.

NGUYEN T.-D., La guerre de l'eau aura-t-elle lieu ? Éditions Johanet, Paris, France, 2004.

OUAZZANI S., DUGELAY E. et TUTENUIT C., L'entreprise et l'eau. Vers une gestion responsable, Étude Epe - Deloitte, Paris, France, 2012.

SCHIFF M., Un cas de censure dans la science : l'affaire de la mémoire de l'eau, Albin Michel, Paris, France, 2004.

TNS-SOFRES \& CIEAU, Les Français et l'eau, Baromètre 2012, $17^{\mathrm{e}}$ édition, Paris, France, 2012.

TREPOS J. Y., Sociologie de l'expertise, Paris, PUF, 1996.

TROGER K., Clés méthodologiques de la socio-anthropologie de l'environnement. Comment aborder la dimension sociale de l'eau, Agence de l'eau, France, 2010.

\section{Articles}

COANUS T., COMBY J. et DUCHÊNE F., « Risques et territoires : interroger et comprendre la dimension locale de quelques risques contemporains », dans Sciences $d u$ risque et du danger, Série Références, 2010.

GRAMAGLIA C., «Des poissons aux masses d'eau : les usages militants du droit pour faire entendre la parole d'êtres qui ne parlent pas ", dans Politix, n 83, 2008, p. 133153.

JONES P.-Y., " Parole d'experts, public profane ", dans Questions de communication, $\mathrm{n}^{\circ} 4,2013$.

KERMISCH C., "Vers une définition multidimensionnelle du risque », dans VertigO - la revue électronique en sciences de l'environnement, volume 12, numéro 2, septembre 2012.

WOLTON D. (dir), «Les chercheurs au cœur de l'expertise », dans Hermès, numéro 64, novembre 2012. 
Résumé : Scandales sanitaires récurrents, les controverses sur la qualité de l'eau de boisson sont inscrites dans le paysage médiatique français. Ces polémiques bâtissent des discours sur la notion de risque. Experts, médias et entreprises interpellent le consommateur autour de sa consommation d'eau à boire jetant le trouble dans ses valeurs et l'invitant à choisir une manière de boire comme une manière d'être.

Mots-clés : discours, perceptions, expertises, espace public, eau de boisson, controverses.

Abstract: Recurring disputes, wars over drinking water are part of the global media agenda. In France, they represent a regular topic of division. Such debates are building discourses and fears about risk in daily life. Experts, medias and water companies are reaching consumers to engage them about their drinking water habits. They urge the public to choose a way to drink as an important societal value.

Keywords: discourse, perceptions, expertise, public sphere, drinking water, disputes. 
